\title{
Semiclassical Calculation of Activation Parameters for Elementary Steps in Catalytic Olefin Hydrogenation in Liquid Media
}

\author{
P. SØGAARD-ANDERSEN and J. ULSTRUP
}

Chemistry Department A, Building 207, The Technical University of Denmark, DK-2800 Lyngby, Denmark

We have applied general electron and atom group transfer theory to calculate activation energies and pre-exponential nuclear tunnelling factors for the oxidative addition and insertion steps in olefin hydrogenation catalyzed by lowvalent rhodium complexes. Nuclear tunnelling is important for bond stretching in the hydrogen molecule, in $\mathrm{Rh}-\mathrm{H}$, and in $\mathrm{C}-\mathrm{C}$ bonds, while other modes contribute fully to the activation energy. Explicit activation parameter expressions in terms of structural and thermodynamic properties of the complexes can be obtained when the nuclear motion is represented by harmonic potentials, and rate parameters calculated on the basis of available data are found to be similar for oxidative addition and insertion.

Calculation of activation energies for elementary steps (dissociation, addition, insertion etc.) in catalytic processes by quantum chemical methods, is presently attracting much attention. ${ }^{1-}$ ${ }^{13}$ Frequently, motion of a few molecular fragments is considered, while synchronous motion of other fragments and the solvent has to be disregarded. It is, however, necessary to provide a prescription for incorporating all molecular and solvent modes. In addition, attention should be given to nuclear tunnelling for the transfer of hydrogen atoms and other small groups. In this work we shall show how this can be achieved by means of recent theoretical formulations of electron and atom group transfer in condensed media. ${ }^{14-17}$ More specifically, we shall calculate activation energies and nuclear tunnelling factors for representative models of elementary steps in the hydrogenation of olefins catalyzed by $\mathrm{RhClL}_{3}$
( $\mathrm{L}=$ triphenylphosphine). This approach can be complemented by representing the molecular modes by nuclear potentials obtained by quantum chemical methods.

\section{PROCEDURE FOR THE CALCULATION OF ACTIVATION PARAMETERS}

We introduce potential energy surfaces for the initial (i) and final (f) states

$U_{\mathrm{i}}=U_{\mathrm{i}}\left(\left\{\mathrm{Q}_{\mathrm{i}}\right\},\{\mathrm{q}\}\right) ; U_{\mathrm{f}}=U_{\mathrm{f}}\left(\left\{\mathrm{Q}_{\mathrm{f}}\right\},\{\mathrm{q}\}\right)+\Delta U_{\mathrm{o}}$

where $\left\{Q_{i}\right\}$ and $\left\{Q_{f}\right\}$ are sets of intramolecular coordinates, $\{\mathrm{q}\}$ the "collective" solvent coordinates, and $\Delta U_{\mathrm{o}}$ the difference between the minima of the surfaces. In general $\left\{Q_{f}\right\}$ are related to $\left\{Q_{i}\right\}$ by a linear transformation, i.e.

$\mathrm{Q}_{\mathrm{f}}^{\mathrm{k}}=\sum_{\mathrm{j}=1}^{\mathrm{N}} \alpha_{\mathrm{kj}} \mathrm{Q}_{\mathrm{i}}^{\mathrm{j}}$

where the superscripts refer to components of $\left\{Q_{i}\right\}$ and $\left\{Q_{f}\right\}, N$ is the number of molecular degrees of freedom, and $\left\{\alpha_{\mathbf{k j}}\right\}$ a transformation matrix. ${ }^{14,15}$ In most cases the solvent can be regarded as "linear". This implies that $\{\mathbf{q}\}$ span harmonic potentials subject to equilibrium shifts only. All coordinates for most organic solvents at room temperature are furthermore classical, ${ }^{18}$ as are most intramolecular modes, with stretching and deformation of bound hydrogen and $\mathrm{C}-\mathrm{C}$ stretching as exceptions.

When the electron exchange integral between 
the zero order states is small the rate probability per unit time takes the form ${ }^{15}$

$W=A \exp \left[-H\left(\theta^{*} ;\left\{\mathrm{Q}_{\mathrm{i}}^{*}\right\},\left\{\mathrm{q}^{*}\right\} ; \Delta U_{\mathrm{o}}\right] \approx\right.$

$A \exp \left[-\sigma\left(\left\{\mathrm{Q}_{\mathrm{iq}}^{*}\right\}\right)\right] \times$

$\exp \left[-U_{\mathrm{i}}^{*}\left(\left\{\mathrm{Q}_{\mathrm{ic}}^{*}\right\},\left\{\mathrm{Q}_{\mathrm{iq}}=0\right\},\left\{\mathrm{q}^{*}\right\} ; \Delta U_{\mathrm{o}}\right) / k_{\mathrm{B}} T\right]$

$A$ is a constant which depends on the topology of the surfaces close to the intersection region and represents the activation entropy, $k_{\mathrm{B}}$ is Boltzmann's constant and $T$ the absolute temperature. The strongest dependence on the structural and thermodynamic parameters is, however, reflected in the quantities $\sigma$ and $U_{\mathrm{i}}^{*}$. The activation energy $U_{\mathrm{i}}^{*}$ is the energy at the saddle point of the intersection between the "classical" parts of $U_{\mathrm{i}}$ and $U_{\mathrm{f}}$, the coordinates of which, $\left\{\mathrm{Q}_{\mathrm{ic}}^{*}\right\}$ and $\left\{Q_{\mathrm{fc}}^{*}\right\}$, are determined by the equations

$$
\left(1-\theta^{*}\right) \frac{\partial U_{\mathrm{i}}}{\partial Q_{\mathrm{ic}}^{j}}+\theta^{*} \frac{\partial U_{\mathrm{f}}}{\partial Q_{\mathrm{ic}}^{j}}=0
$$

$U_{\mathrm{i}}\left(\left\{\mathrm{Q}_{\mathrm{ic}}^{*}\right\},\left\{\mathrm{Q}_{\mathrm{iq}}=0\right\},\left\{\mathrm{q}^{*}\right\}\right)=$

$U_{\mathrm{f}}\left(\left\{\mathrm{Q}_{\mathrm{fc}}^{*}\right\},\left\{\mathrm{Q}_{\mathrm{iq}}=0\right\},\left\{\mathrm{q}^{*}\right\}\right)+\Delta U_{\mathrm{o}}$

where $\theta^{*}$ is the transfer coefficient, $\theta^{*}=\partial U_{\mathrm{i}}$ $\left(\left\{\mathbf{Q}_{\mathrm{i}}^{*}\right\},\left\{\mathrm{q}^{*}\right\}\right) / \partial \Delta U_{\mathrm{o}}$.

The high-frequency coordinates $\left\{Q_{\mathrm{iq}}\right\}$ (the equilibrium values are taken as zero initially and as $\left\{Q_{\text {fqo }}\right\}$ in the final state) determine the temperature independent nuclear tunnelling factor $\sigma\left(\left\{\mathbf{Q}_{\mathrm{iq}}^{*}\right\}\right)$. Although interconversion between high-frequency quantum and low-frequency classical modes can be handled generally, we use here the harmonic approximation which provides compact rate expressions and is at the same time a reasonable approximation for the modes in question here ( $c f$. , however, section 5). For $\left\{\mathrm{Q}_{\mathrm{iq}}\right\}$ eqn. (3) must then be replaced by the following equation ${ }^{15}$

$Q_{\mathrm{iq}}^{*} \operatorname{th}\left[\beta \hbar \Omega_{\lambda}\left(1-\theta^{*}\right)\right] / 2+$

$\left.\sum_{\rho} \tau_{\rho \lambda}\left(Q_{\mathrm{pq}}^{\rho^{*}}-Q_{\mathrm{qo}}^{\rho}\right) t h \beta \hbar \Omega_{\rho} \theta^{*}\right] / 2=0$

where $Q_{\mathrm{iq}}^{\lambda}$ are the high-frequency coordinates in the initial state which are converted to the final state coordinates $\mathrm{Q}_{\mathrm{fq}}$ by the transformation matrix $\left\{\tau_{\rho \lambda}\right\}, \Omega_{\lambda}$ and $\Omega_{\rho}$ are vibrational frequencies and $\hbar$ Planck's constant divided by $2 \pi$.

If the exchange integral is not small, the activation energy is given by the maximum of the lowest adiabatic surface. ${ }^{14}$ For atom group transfer it can be expected that this modification is frequently necessary, but we shall disregard it in the present approach.

\section{ELEMENTARY STEPS IN CATALYTIC HYDROGENATION}

The sequence of steps in Scheme 1 has emerged from many investigations. ${ }^{19,20}$

Structural data are only available for the original catalyst molecule (i). A T-shaped structure for $\mathrm{RhL}_{3}^{+}$, presumably similar to that of $\mathrm{RhClL}_{2}$, has been reported, ${ }^{21}$ and SCF calculations suggest that the two possible ligand arrangements in a $\mathrm{T}$-shaped structure for $\mathrm{RhClL}_{2}$ are about equally stable. ${ }^{12 a}$ NMR data suggest that the structure of (iii) is III in Fig. 1. ${ }^{22,23}$ This is borne out by isolated complexes containing bulkier phosphine ligands ${ }^{24,25}$ and by SCF calculations. ${ }^{12 a}$ Steps involving this intermediate could furthermore be solvent dependent due to the vacant coordination site. The existence of (iv) (i) $\mathrm{RhClL}_{3}$

(ii) $\mathrm{RhClL}_{2}+\mathrm{H}_{2}$

(iii) $\mathrm{H}_{2} \mathrm{RhClL}_{2}+$ olefin

(iv) $\mathrm{H}_{2} \mathrm{RhClL}_{2}$ (olefin)

(v) $\mathrm{HRhClL}_{2}$ (alkyl)

$$
\rightarrow \mathrm{RhClL}_{2}+\mathrm{L}
$$$$
\rightarrow \mathrm{H}_{2} \mathrm{RhClL}_{2}
$$$$
\rightarrow \mathrm{H}_{2} \mathrm{RhClL}_{2} \text { (olefin) }
$$$$
\rightarrow \mathrm{HRhClL}_{2} \text { (alkyl) }
$$$$
\rightarrow \mathrm{RhClL}_{2}+\text { alkane }
$$

Dissociation

Oxidative Addition

Association

Insertion

Reductive Elimination

\section{Scheme 1.}




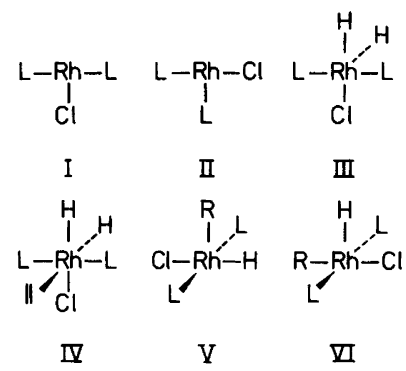

Fig. 1. Isomers of intermediates in $\mathrm{RhClL}_{3}$ catalyzed olefin hydrogenation.

and $(v)$ has been inferred from kinetic data. SCF calculations indicate that among isomers which originate from III with little molecular reorganization energy expenditure, IV is significantly more stable than other isomers. ${ }^{12 a}$ The most stable isomers of $(\mathrm{v})$ are finally $\mathrm{V}$ and VI. ${ }^{12}$ Formation of $\mathrm{V}$ from IV requires, however, far less intramolecular activation energy than conversion between other energetically accessible isomers. In the following we therefore restrict our considerations to I, III, IV and V.

\section{ACTIVATION PARAMETER EXPRESSIONS}

The rate determining step for $\mathrm{RhClL}_{3}$-catalyzed hydrogenation of ethylene is believed to be insertion, ${ }^{19,20}$ but either this step or oxidative addition could conceivably be rate determining for different olefins, catalysts or solvents. We therefore derive activation parameters for the four steps (6)-(9).

(A) Dissociation and association. The potential energy surfaces for dissociation are

$$
\begin{aligned}
& U_{\mathrm{i}}=v_{\mathrm{i}}\left(\mathrm{Q}_{\mathrm{L}}\right)+\frac{1}{2} \sum_{j=1}^{N-1} M_{\mathrm{j}} \Omega_{\mathrm{j}}^{2} Q_{\mathrm{j}}^{2}+\frac{1}{2} \sum_{\varkappa} \hbar \omega_{\varkappa} q_{\varkappa}^{2} \\
& U_{\mathrm{f}}=u_{\mathrm{f}}\left(\mathrm{Q}_{\mathrm{L}}\right)+\frac{1}{2} \sum_{j=1}^{N-1} M_{\mathrm{j}} \Omega_{\mathrm{j}}^{2}\left(Q_{\mathrm{j}}-Q_{\mathrm{jo}}\right)^{2}+ \\
& \frac{1}{2} \sum_{\varkappa} \hbar \omega_{\varkappa}\left(q_{\varkappa}-q_{\varkappa \mathrm{o}}\right)^{2}+\Delta U_{\mathrm{o}}
\end{aligned}
$$

The first term represents the outgoing ligand, the second ones the other ligands, and the third terms the solvent, where $q_{\kappa}$ and $\omega_{\kappa}$ are (dimensionless) coordinates and vibrational frequencies. Equilibrium values for all coordinates have been taken as zero initially, while the zero subscripts refer to the final state. The coordinates $Q_{\mathrm{j}}$ (taken as identical for $U_{\mathrm{i}}$ and $U_{\mathrm{f}}$ ) are represented by the harmonic approximation in view of their small shifts. $M_{\mathrm{j}}$ are effective masses, and the vibrational frequencies $\Omega_{\mathrm{j}}$ are related to the actual frequencies in the initial and final states, $\Omega_{j}^{(i)}$ and $\Omega_{j}^{(\mathrm{f})}$, by the equation

$\Omega_{\mathrm{j}}=2 \Omega_{\mathrm{j}}^{(\mathrm{i})} \Omega_{\mathrm{j}}^{(\mathrm{f})} /\left(\Omega_{\mathrm{j}}^{(\mathrm{i})}+\Omega_{\mathrm{j}}^{(\mathrm{f})}\right)$.

$Q_{\mathrm{L}}$ represents initially a bound state. In the final state $\mathrm{L}$ is weakly bound in a solvent cage. As a consequence $\partial v_{\mathrm{i}} / \partial Q_{\mathrm{L}}>>\partial u_{\mathrm{f}} / \partial Q_{\mathrm{L}}$, and provided that $\theta^{*}$ is not close to zero or unity, eqn. (3) shows that $Q_{\mathrm{L}}^{*}$ practically coincides with the initial equilibrium value of $Q_{\mathrm{L}} \cdot{ }^{26,27} \mathrm{Eqn}$. (4) then gives

$\theta^{*}=\frac{1}{2}+\frac{\Delta U_{\mathrm{o}}+E_{\mathrm{rL}}^{\mathrm{f}}}{2\left(E_{\mathrm{s}}+E_{\mathrm{r}}\right)} ;$

$E_{\mathrm{A}}=\left(E_{\mathrm{s}}+E_{\mathrm{r}}+E_{\mathrm{rL}}^{\mathrm{f}}+\Delta U_{\mathrm{o}}\right)^{2} / 4\left(E_{\mathrm{s}}+E_{\mathrm{r}}\right)$

where we have introduced reorganization energies for the solvent, $E_{\mathrm{s}}=\frac{1}{2} \sum_{x} \hbar \omega_{x} q^{2}{ }_{x o}$, the molecular modes, $E_{\mathrm{r}}=\frac{1}{2} \sum_{j=1}^{N-1} M_{\mathrm{j}} \Omega_{\mathrm{j}}^{2} Q_{\mathrm{jo}}^{2}$, and the outgoing ligand, $E_{\mathrm{rL}}^{\mathrm{f}}=u_{\mathrm{f}}\left(Q_{\mathrm{L}}=\mathrm{O}\right)$.

A similar calculation gives for association ${ }^{26,27}$

$\theta^{*}=\frac{1}{2}+\frac{\Delta U_{\mathrm{o}}-E_{\mathrm{rc}}^{\mathrm{i}}}{2\left(E_{\mathrm{s}}+E_{\mathrm{r}}\right)}$

$E_{\mathrm{A}}=\left(E_{\mathrm{s}}+E_{\mathrm{r}}+\Delta U_{\mathrm{o}}-E_{\mathrm{rc}}^{\mathrm{i}}\right)^{2} / 4\left(E_{\mathrm{s}}+E_{\mathrm{r}}\right)$

where $E_{\mathrm{rC}}^{\mathrm{i}}=v_{\mathrm{i}}\left(Q_{\mathrm{L}}=Q_{\mathrm{LO}}\right)$ is the reorganization energy for the incoming substrate molecule.

(B) Oxidative addition. We represent the solvent and non-hydride ligands as above. However, the state of the hydrogen atoms for cis-addition - which we consider here - changes drastically. Initially there are three hindered translational and two hindered rotational degrees of freedom largely involving $\mathrm{H}_{2}$, and one stretching mode of this molecule. In the final state these 


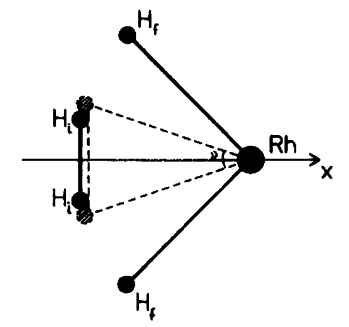

Fig. 2. Oxidative addition. The fully drawn lines indicate the equilibrium configuration of the $\mathrm{H}_{2}$ molecule at the transition configuration for the coordinate $\mathrm{x}$, and the equilibrium configuration of the dihydrido complex. The hydrogen atoms in these two states are characterized by the subscripts $i$ (initial) and f (final), respectively. The filled circles show the positions of the hydrogen and rhodium atoms. In the transition configuration, shown by the dashed lines, the $\mathrm{H}_{2}$ bond is less distorted than the $\mathrm{H}-\mathrm{Rh}-\mathrm{H}$ bending mode.

modes are converted to a symmetric and an asymmetric stretching, a bending mode of the $\mathrm{H}-\mathrm{Rh}-\mathrm{H}$ unit and three orientational modes of the complex. Secondly, $\mathrm{H}_{2}$ stretching is a highfrequency quantum mode, ${ }^{14-17}$ while other initial state modes are classical. In the final state $\mathrm{Rh}-\mathrm{H}$ stretchings are of quantum nature, the bending mode in the intermediate range, while the orientational modes are classical. cis-Addition of $\mathrm{H}_{2}$ thus involves both "mixing" and "interconversion" of quantum and classical modes.

We consider approach of $\mathrm{H}_{2}$ to $\mathrm{RhClL}_{2}$ in a plane perpendicular to the $\mathrm{L}-\mathrm{Rh}-\mathrm{L}$ axis. The orientation shown in Fig. 2 requires the smallest reorganization, but different configurations could be suitably averaged. In this figure the chlorine ligand is thus in trans position with respect to one of the hydride ligands $\mathrm{H}_{\mathrm{f}}$, and the $\mathrm{Rh}-\mathrm{L}$ bonds perpendicular to the plane shown. We then only need to consider the following simplified potential energy expression for the initial state

$$
\begin{aligned}
& U_{\mathrm{i}}=\frac{1}{2}\left(2 M_{\mathrm{H}}\right) \Omega_{\mathrm{x}}^{2} \mathrm{Q}_{\mathrm{x}}^{2}+\frac{1}{2}\left(\frac{1}{2} M_{\mathrm{H}}\right) \Omega_{\mathrm{i}}^{\mathrm{s} 2} \mathrm{Q}_{\mathrm{i}}^{\mathrm{s} 2}+ \\
& \frac{1}{2} \sum_{\mathrm{j}=1} M_{\mathrm{j}} \Omega_{\mathrm{j}}^{2} \mathrm{Q}_{\mathrm{j}}^{2}+\frac{1}{2} \sum_{x} h \omega_{x} q_{x}^{2}
\end{aligned}
$$

The first term represents hindered translation of $\mathrm{H}_{2}$ along the $x$ axis, the second one stretching of $\mathrm{H}_{2}$, the $\Omega$ 's are vibrational frequencies, and $M_{\mathrm{H}}$ the mass of a hydrogen atom. The potential energy of the final state is

$$
\begin{aligned}
& U_{\mathrm{f}}=\frac{1}{2}\left(2 M_{\mathrm{H}}\right) \Omega_{\mathrm{f}}^{\mathrm{s} 2}\left(Q_{\mathrm{f}}^{\mathrm{s}}-Q_{\mathrm{fo}}^{\mathrm{s}}\right)^{2}+ \\
& \frac{1}{2}\left(2 M_{\mathrm{H}}\right) \Omega_{\mathrm{f}}^{\mathrm{b} 2}\left(Q_{\mathrm{f}}^{\mathrm{b}}-Q_{\mathrm{fo}}^{\mathrm{b}}\right)^{2}+\frac{1}{2} \sum_{\mathrm{j}=1}^{\mathrm{N}^{\prime}} M_{\mathrm{j}} \Omega_{\mathrm{j}}^{2}\left(Q_{\mathrm{j}}-Q_{\mathrm{jo}}\right)^{2}+ \\
& \frac{1}{2} \sum_{\varkappa} \hbar \omega_{\varkappa}\left(q_{\varkappa}-q_{\kappa \mathrm{o}}\right)^{2}+\Delta U_{\mathrm{o}}
\end{aligned}
$$

where the first two terms now refer to symmetric stretching (s) and bending (b). The relation between the "mixed" coordinates is

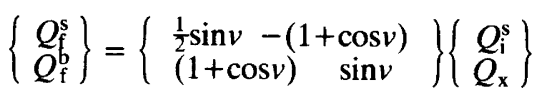

where $v$ is the angle of the $\mathrm{H}-\mathrm{Rh}-\mathrm{H}$ unit close to the transition configuration.

Eqns. (3)-(5) and (15) - (17) give the following coordinates for the transition configuration

$$
q_{x}^{*}=\theta^{*} q_{\text {кo }} ; \quad Q_{\mathrm{j}}^{*}=\theta^{*} Q_{\mathrm{jo}}
$$

$$
Q_{\mathrm{j}}^{\mathrm{s}^{*}} \approx
$$

$$
Q_{\mathrm{io}}^{\mathrm{s}}-Q_{\mathrm{io}}^{\mathrm{s}} /\left[1+(1+\cos v)^{2}\left(\Omega_{\mathrm{f}}^{\mathrm{b}} \Omega_{\mathrm{f}}^{\mathrm{s}} / \Omega_{\mathrm{i}}^{\mathrm{s} 2}\right)^{1 / 2} \text { th } \frac{\beta \hbar \Omega_{\mathrm{f}}^{\mathrm{b}} \theta^{*}}{2}\right]
$$

$$
\begin{aligned}
& Q_{\mathrm{x}}^{*}-Q_{\mathrm{xo}} \approx \\
& \frac{1}{2}[\sin v /(1+\cos v)]\left[1-\left(\Omega_{\mathrm{f}}^{\mathrm{b}} / \Omega_{\mathrm{f}}^{\mathrm{s}}\right) \operatorname{th} \beta \hbar \Omega_{\mathrm{f}}^{\mathrm{b}} \theta^{*} / 2\right] \times \\
& \left(Q_{\mathrm{i}}^{\mathrm{s}^{*}}-Q_{\mathrm{io}}^{\mathrm{s}}\right) \\
& Q_{\mathrm{f}}^{\mathrm{b}^{*}}-Q_{\mathrm{fo}}^{\mathrm{b}} \approx(1+\cos v)\left(Q_{\mathrm{i}}^{\mathrm{s}^{*}}-Q_{\mathrm{io}}^{\mathrm{s}}\right)
\end{aligned}
$$

$Q_{\mathrm{f}}^{\mathrm{s}^{*}}-Q_{\mathrm{fo}}^{\mathrm{s}} \approx \frac{1}{2} \sin v\left(\Omega_{\mathrm{f}}^{\mathrm{b}} / \Omega_{\mathrm{f}}^{\mathrm{s}}\right)$ th $\frac{\beta \hbar \Omega_{\mathrm{f}}^{\mathrm{b}} \theta^{*}}{2}\left(Q_{\mathrm{i}}^{\mathrm{s}^{*}}-Q_{\mathrm{io}}^{\mathrm{s}}\right)$

where $Q_{\mathrm{xo}}$ and $Q_{\mathrm{io}}^{\mathrm{s}}$ are the equilibrium values of $Q_{\mathrm{x}}$ and $Q_{\mathrm{i}}^{\mathrm{s}}$ in the final state (we have here ignored $\sin ^{2} v$ compared with $(1+\cos v)^{2}$ close to the saddle point where $Q_{f}^{b}$ is significantly distorted). These equations suggest the following sequence of events. The classical modes, i.e. those of the solvent, the $\mathrm{Rh}-\mathrm{Cl}$ and $\mathrm{Rh}-\mathrm{L}$ modes, and $x$ are 
first reorganized to the configuration given by eqns. (18) and (20), providing most of the activation energy. This is followed by $\mathrm{H}_{2}$ bond stretch by nuclear tunnelling to the configuration given by eqn. (19). For reported or suggested frequency values ${ }^{22-25}\left(\Omega_{\mathrm{f}}^{\mathrm{b}} \approx 600-1000 \mathrm{~cm}^{-1}, \Omega_{\mathrm{f}}^{\mathrm{s}}\right.$ $\approx 2000 \mathrm{~cm}^{-1}$ and $\left.\Omega_{\mathrm{i}}^{6} \approx 4000 \mathrm{~cm}^{-1}\right)$ and $\theta^{*} \approx 0.1-0.3$ (cf. the concluding section) it amounts to $15-30 \%$ of the total shift of $Q_{i}^{s}$. Subsequent to changes of the electronic structure the dihydrido complex is formed, with instantaneous $Q_{\mathrm{f}}^{\mathrm{s}}$ and $Q_{\mathrm{f}}^{\mathrm{b}}$ values given by eqns. (21) and (22). $Q_{\mathrm{f}}^{\mathrm{b}}$ is much more vibrationally excited than $Q_{f}^{s}$ which is shifted by nuclear tunnelling to its vibrational ground level.

The exponent $H$ in eqn. (3) is now found by solving the following equation with respect to $\theta^{*}$

$$
\begin{aligned}
& \left(2 \theta^{*}-1\right)\left(E_{\mathrm{s}}+E_{\mathrm{r}}\right)+\frac{1}{2}\left(2 M_{\mathrm{H}}\right) \Omega_{\mathrm{x}}^{2} Q_{\mathrm{x}}^{* 2}= \\
& \frac{1}{2}\left(2 M_{\mathrm{H}}\right) \Omega_{\mathrm{f}}^{\mathrm{b}}\left(Q_{\mathrm{f}}^{\mathrm{b}^{*}}-Q_{\mathrm{fo}}^{\mathrm{b}}\right) c h^{-2}\left(\beta \mathrm{th} \Omega_{\mathrm{f}}^{\mathrm{b}} \theta^{*} / 2\right)+\Delta U_{\mathrm{o}}
\end{aligned}
$$

where the explicit dependence of $Q_{\mathrm{x}}^{*}$ and $Q_{\mathrm{f}}^{\mathrm{b}}$ on $\theta^{*}$ is given by eqns. (20) and (21). This equation can be handled numerically, and the resulting $\theta^{*}$ inserted in eqns. (1) and (3) to give the activation energy and tunnelling factor. Analytical expressions can, however, be obtained in the following two limiting cases:

(1) For $\beta \hbar \Omega_{\mathrm{f}}^{\mathrm{b}} \theta^{*} / 2>>1$, corresponding to a high bending frequency. The activation energy and the nuclear tunnelling factor are then

$$
\begin{aligned}
& E_{\mathrm{A}}=E_{\mathrm{rh}}^{\mathrm{x}}+\left(E_{\mathrm{s}}+E_{\mathrm{r}}+\Delta U_{\mathrm{o}}-E_{\mathrm{rh}}^{\mathrm{x}}\right)^{2} / 4\left(E_{\mathrm{s}}+E_{\mathrm{r}}\right) \\
& \sigma \approx \frac{1}{2}\left(M_{\mathrm{H}} / \hbar\right)(1+\cos v)^{2} \times \\
& \frac{4\left(\Omega_{\mathrm{f}}^{\mathrm{b}} / \Omega_{\mathrm{f}}^{\mathrm{q}}\right)+\left(\Omega_{\mathrm{f}}^{\mathrm{b}} / \Omega_{\mathrm{i}}^{\mathrm{s}}\right)(1+\cos v)^{2}}{\left[1+(1+\cos v)^{2}\left(\Omega_{\mathrm{q}}^{\mathrm{b}} \Omega_{\mathrm{f}}^{\mathrm{s}} / \Omega_{\mathrm{i}}^{\mathrm{s} 2}\right)^{1 / 2}\right]^{2}} Q_{\mathrm{io}}^{\mathrm{s}}
\end{aligned}
$$

where $E_{\mathrm{rh}}^{\mathrm{x}}$ is the reorganization energy for $\mathrm{H}_{2}$ motion along the coordinate $x$, from its initial equilibrium position to the position given by eqn. (20) with $\operatorname{th}\left(\beta \hbar \Omega_{\mathrm{f}}^{\mathrm{b}} \theta^{*} / 2\right)$ replaced by unity.

(2) In the opposite limit $\beta \hbar \Omega_{\mathrm{f}}^{\mathrm{b}} \boldsymbol{\theta}^{*} / 2<<1$. The tunnelling factor now vanishes, while $E_{\mathrm{A}}$ is

$$
E_{\mathrm{A}}=\left(E_{\mathrm{s}}+E_{\mathrm{r}}+E_{\mathrm{r}}^{\mathrm{b}}+\Delta U_{\mathrm{o}}-E_{\mathrm{r} l}^{\mathrm{x}}\right)^{2} / 4\left(E_{\mathrm{s}}+E_{\mathrm{r}}\right)
$$

$E_{\mathrm{r}}^{\mathrm{b}}$ is the energy required to distort $Q_{\mathrm{f}}^{\mathrm{b}}$ - at fixed $Q_{\mathrm{f}}^{\mathrm{s}}$ identical to its final equilibrium value - from final state equilibrium to the value which corresponds to a distance between the two hydrogen. atoms identical to the equilibrium bond length of the $\mathrm{H}_{2}$ molecule. $E_{\mathrm{rl}}^{\mathrm{x}}$ is the energy required to bring an $\mathrm{H}_{2}$ molecule from its initial equilibrium location, along $x$, to the position which corresponds to such a deformation of $Q_{\mathrm{f}}^{\mathrm{b}}$.

(C) Insertion. This step is viewed as planar motion of coordinated ethylene and a cis-located hydride ligand (Fig. 3). We again only include coordinates subject to equilibrium shifts. The initial state potential energy is then

$$
\begin{aligned}
& U_{\mathrm{i}}=\frac{1}{2} M_{\mathrm{et}} \Omega_{\mathrm{is}}^{\mathrm{et} 2} Q_{\mathrm{s}}^{\mathrm{e} 2}+\frac{1}{2} \mu_{\mathrm{et}} \Omega_{\mathrm{is}}^{\mathrm{cc} 2} Q_{\mathrm{is}}^{\mathrm{cc} 2}+ \\
& \frac{1}{2} M_{\mathrm{et}} \Omega_{\mathrm{b}}^{\mathrm{et} 2} Q_{\mathrm{b}}^{\mathrm{et} 2}+\frac{1}{2} \mu_{\mathrm{et}}^{\mathrm{i}} \Omega_{\mathrm{ib}}^{\mathrm{cc} 2} Q_{\mathrm{ib}}^{\mathrm{cc} 2}+ \\
& \frac{1}{2} M_{\mathrm{H}} \Omega_{\mathrm{is}}^{\mathrm{H} 2} Q_{\mathrm{is}}^{\mathrm{H} 2}+\frac{1}{2} M_{\mathrm{H}} \Omega_{\mathrm{ib}}^{\mathrm{H} 2} Q_{\mathrm{ib}}^{\mathrm{H} 2}+ \\
& \frac{1}{2} \sum_{\mathrm{j}=\mathrm{i}}^{N^{\prime}} M_{\mathrm{j}} \Omega_{\mathrm{j}}^{2} Q_{\mathrm{j}}^{2}+\frac{1}{2} \sum_{\varkappa} \hbar \omega_{\varkappa} q_{\varkappa}^{2}
\end{aligned}
$$

The first two terms represent stretching and bending (the angle $\beta$ in Fig. 3) of the bond between $\mathrm{Rh}$ and the mass centre of ethylene (with $\Omega$ 's referring to vibrational frequencies and $M$ 's and $\mu$ 's to masses or reduced masses), the third term stretching of the olefin $\mathrm{C}-\mathrm{C}$ bond, and the fourth bending of ethylene around the angle $\chi$. The fifth and sixth terms refer to stretching and bending of the $\mathrm{Rh}-\mathrm{H}$ bond, and the last two terms have the same meaning as before. All modes except $\mathrm{C}-\mathrm{C}$ stretching and those involving the $\mathrm{Rh}-\mathrm{H}$ bond can furthermore be regarded as classical.

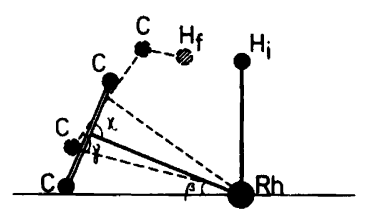

Fig. 3. Insertion. The fully drawn lines show the initial state equilibrium configuration of coordinated ethylene and hydrogen $\left(\mathrm{H}_{\mathrm{i}}\right)$. The dashed line shows the transition configuration formed by motion along the angle $\beta$ and providing the shortest possible distance for hydrogen atom transfer. The position of the hydrogen atom after transfer is also indicated $\left(\mathrm{H}_{\mathrm{f}}\right)$. This distance would be shortened by compression of the Rh-ethylene bond and twisting around the angle $\chi$. 

is

The potential energy surface for the final state

$U_{\mathrm{f}}=\frac{1}{2} M_{\mathrm{et}} \Omega_{\mathrm{fs}}^{\mathrm{et} 2}\left(Q_{\mathrm{s}}^{\mathrm{et}}-Q_{\mathrm{so}}^{\mathrm{et}}\right)^{2}+\frac{1}{2} M_{\mathrm{et}} \Omega_{\mathrm{fb}}^{\mathrm{et} 2}\left(Q_{\mathrm{b}}^{\mathrm{et}}-Q_{\mathrm{bo}}^{\mathrm{et}}\right)^{2}+$

$\frac{1}{2} \mu_{\mathrm{et}} \Omega_{\mathrm{fs}}^{\mathrm{cc} 2}\left(Q_{\mathrm{s}}^{\mathrm{cc}}-Q_{\mathrm{so}}^{\mathrm{cc}}\right)^{2}+\frac{1}{2} \mu_{\mathrm{et}}^{\mathrm{f}} \Omega_{\mathrm{fb}}^{\mathrm{cc} 2}\left(Q_{\mathrm{fb}}^{\mathrm{cc}}-Q_{\mathrm{fbo}}^{\mathrm{cc}}\right)^{2}+$

$\frac{1}{2} M_{\mathrm{u}} \Omega_{\mathrm{fb}}^{\mathrm{H} 2}\left(Q_{\mathrm{is}}^{\mathrm{H}}-Q_{\mathrm{iso}}^{\mathrm{H}}\right)^{2}+\frac{1}{2} M_{\mathrm{H}} \Omega_{\mathrm{fs}}^{\mathrm{H}}\left(Q_{\mathrm{ib}}^{\mathrm{H}}-Q_{\mathrm{ibo}}^{\mathrm{H}}\right)^{2}+$

$\frac{1}{2} \sum_{j=1}^{N^{\prime}} M_{\mathrm{j}} \Omega_{\mathrm{j}}^{2}\left(Q_{\mathrm{j}}-Q_{\mathrm{jo}}\right)^{2}+\frac{1}{2} \sum_{x} \hbar \omega_{x}\left(q_{x}-q_{x \mathrm{o}}\right)^{2}+\Delta U_{\mathrm{o}}$

Stretching and bending of the bond between $\mathrm{Rh}$ and the mass centre of coordinated alkyl, and $\mathrm{C}-\mathrm{C}$ stretching (the first three terms in eqn. (28) are given by the same coordinates as for the initial state. The hydride ligand is converted to a bound state at the $\beta$-carbon atom. The transition configuration favours an orientation where this motion is conversion of $\mathrm{Rh}-\mathrm{H}$ bending to $\mathrm{C}-\mathrm{H}$ stretching and of $\mathrm{Rh}-\mathrm{H}$ stretching to $\mathrm{C}-\mathrm{H}$ bending (the fifth and sixth terms). This path could be generalized to incorporate the remaining hydrogen atoms at carbon. The hybridization change thus causes equilibrium change in the $\mathrm{C}-\mathrm{H}$ bending modes in the plane, but this is of minor importance compared with reorganization in other parts of the molecule. The fourth term refers to bending of the $\mathrm{Rh}-\mathrm{C}-\mathrm{C}$ unit (the angle $\gamma$ ). For small fluctuations this coordinate is "proportional" to $Q_{\mathrm{ib}}^{\mathrm{cc}}$ (bending at the angle $\chi$ ), so that the coordinate change essentially involves equilibrium and frequency shift.

All classical saddle point coordinates are then given by equations of the same kind as eqn. (18). The following equation is valid for high-frequency $\mathrm{C}-\mathrm{C}$ stretching

$Q_{\mathrm{s}}^{\mathrm{c} *}+\left(\Omega_{\mathrm{fs}}^{\mathrm{cc}} / \Omega_{\mathrm{is}}^{\mathrm{cc}}\right)^{1 / 2}\left(Q_{\mathrm{s}}^{\mathrm{cc} *}-Q_{\mathrm{so}}^{\mathrm{cc}}\right)=0$

where we have put $Q_{\mathrm{ib}}^{\mathrm{cc}}=Q_{\mathrm{fb}}^{\mathrm{cc}}=Q_{\mathrm{b}}^{\mathrm{cc}}$. The effect of this mode is thus reflected solely in the tunnelling factor. The equilibrium geometries of the $\mathrm{Rh}-\mathrm{C}-\mathrm{C}$ framework in the initial and final states corresponds to widely separated positions of the hydrogen atom which is transferred. The hydrogen atom transfer path therefore depends explicitly on the instantaneous position of the $\mathrm{Rh}-\mathrm{C}-\mathrm{C}$ unit. In view of the expected low vibrational frequency of the bending mode of this unit and the high frequencies of the modes involving bound hydrogen, it is a good approximation to regard the coordinates for hydrogen atom transfer as referring to the intermediate configuration for the rest of the molecule, as determined by the effects of repulsive interactions between strongly distorted $\mathrm{Rh}-\mathrm{C}-\mathrm{C}$ and weakly distorted $\mathrm{Rh}-\mathrm{H}$ bonds. Improvement of this point would require that we introduce the explicit dependence of the hydrogen transfer probability on the transfer distance and the parameters of a repulsive potential. The saddle point coordinates for the $\mathrm{Rh}-\mathrm{H}$ are now

$Q_{\text {is }}^{\mathrm{H}^{*}}+\left(\Omega_{\mathrm{fb}}^{\mathrm{H}} / \Omega_{\text {is }}^{\mathrm{H}}\right)^{1 / 2}\left(Q_{\text {is }}^{\mathrm{H}^{*}}-Q_{\text {iso }}^{\mathrm{H}}\right)=0$

$Q_{\mathrm{ib}}^{\mathrm{H}^{*}}$ th $\frac{\beta \hbar \Omega_{\mathrm{ib}}^{\mathrm{H}} \theta^{*}}{2}+\left(\Omega_{\mathrm{fs}}^{\mathrm{H}} / \Omega_{\mathrm{ib}}^{\mathrm{H}}\right)^{1 / 2}\left(Q_{\mathrm{ib}}^{\mathrm{H}^{*}}-Q_{\mathrm{ibo}}^{\mathrm{H}}\right)=0$

$Q_{\mathrm{ib}}^{\mathrm{H}}$ furthermore coincides approximately with $Q_{\text {ibo }}^{\mathrm{H}}\left(\beta \hbar \Omega_{\mathrm{ib}}^{\mathrm{H}} \theta^{*} / 2 \approx 0.3-0.4, c f\right.$. the following section). The activation energy and tunnelling factor are then

$$
\begin{aligned}
& E_{\mathrm{A}} \approx\left(E_{\mathrm{s}}+E_{\mathrm{r}}+E_{\mathrm{r} . \mathrm{st}}^{\mathrm{et}}+E_{\mathrm{r} . \mathrm{b}}^{\mathrm{et}}+E_{\mathrm{r} . \mathrm{b}}^{\mathrm{cc}}+\Delta U_{\mathrm{o}}-E_{\mathrm{r} . \mathrm{b}}^{\mathrm{H}}\right)^{2 /} \\
& 4\left(E_{\mathrm{s}}+E_{\mathrm{r}}+E_{\mathrm{r} . \mathrm{st}}^{\mathrm{et}}+E_{\mathrm{r} . \mathrm{b}}^{\mathrm{cc}}\right) \\
& \sigma \approx \sigma_{\mathrm{s}}^{\mathrm{cc}}+\sigma_{\mathrm{s}}^{\mathrm{H}}
\end{aligned}
$$

where $E_{\mathrm{r}, \mathrm{st}}^{\mathrm{et}}, E_{\mathrm{r}, \mathrm{b}}^{\mathrm{et}}, E_{\mathrm{r}, \mathrm{b}}^{\mathrm{cc}}$ and $E_{\mathrm{r}, \mathrm{b}}^{\mathrm{H}}$ are the reorganization energies for stretching and bending of the $\mathrm{Rh}-\mathrm{C}-\mathrm{C}$ unit, for $\mathrm{C}-\mathrm{C}$ bending, and for $\mathrm{Rh}-\mathrm{H}$ bending, respectively, and $\sigma_{\mathrm{s}}^{\mathrm{cc}}$ and $\sigma_{\mathrm{s}}^{\mathrm{H}}$ the tunnelling contributions from $\mathrm{C}-\mathrm{C}$ and $\mathrm{Rh}-\mathrm{H}$ stretching, i.e.

$\sigma_{\mathrm{s}}^{\mathrm{cc}} \approx\left(\mu_{\mathrm{et}} / \hbar\right) \Omega_{\mathrm{is}}^{\mathrm{cc}} \Omega_{\mathrm{fs}}^{\mathrm{cc}} /\left(\Omega_{\mathrm{is}}^{\mathrm{cc}}+\Omega_{\mathrm{fs}}^{\mathrm{cc}}\right) Q_{\mathrm{so}}^{\mathrm{cc} 2}$

$\sigma_{\mathrm{s}}^{\mathrm{H}} \approx\left(M_{\mathrm{H}} / \hbar\right)\left[\Omega_{\mathrm{is}}^{\mathrm{H}} \Omega_{\mathrm{fb}}^{\mathrm{H}} /\left(\Omega_{\mathrm{is}}^{\mathrm{H}}+\Omega_{\mathrm{fb}}^{\mathrm{H}}\right)\right] Q_{\mathrm{iso}}^{\mathrm{H} 2}$

\section{CONCLUDING REMARKS}

We have provided rate parameter expressions for several steps in catalytic olefin hydrogenation, in terms of rate theory for chemical processes in liquid solutions. ${ }^{14-17}$ This approach is general in the sense that it incorporates all nuclear modes including those of the solvent. It also accounts for both thermal activation and nuclear tunnelling, and for the effects of highand low-frequency mode "mixing" and "interconversion".

In addition to the geometric restrictions inherent in the planar motion shown - which could obviously be relaxed - our most important

Acta Chem. Scand. A 37 (1983) No. 7 
simplifying assumptions are firstly that we have ignored the electron exchange integral and determined the many-dimensional barrier for nuclear motion as the saddle point between two zero order potential energy surfaces, and secondly, application of the harmonic approximation for "mixing" and "interconversion". With these assumptions, rather compact and transparent expressions for the activation energy and nuclear tunnelling factors can be derived. However, the major conclusions are not affected, and numerical treatment of the procedure can be provided if rather general molecular potentials, including potentials calculated by quantum chemical methods, are used. For example, the nuclear wave functions of the high-frequency modes can be combined with the electron exchange integral in an adiabatic approach with respect to separation between low-frequency modes on one hand and the combined electronic and high-frequency nuclear system on the other. ${ }^{28}$ Also, the transition configuration coincides with the equilibrium configuration for the high-frequency state for general molecular potentials, when a particular nuclear mode is converted from a high-frequency to a low-frequency state or vice versa.

The high- and low-frequency modes for the insertion step are separated in the approach which we have taken. From reported experimental values of the activation energy in the range 7-18 $\mathrm{kcal}^{29-31}$ for insertion of cyclohexene and SCF calculations of $\Delta U_{\mathrm{o}}=-17 \mathrm{kcal}$ for the step represented by eqn. $(9)^{12 \mathrm{~b}}$ values of both $\theta^{*}$ and the combined reorganization energy terms can be calculated by means of eqn. (32). We can here disregard the contribution from the $\mathrm{Rh}-\mathrm{H}$ bending mode which is small for the insertion step. The resulting values are thus $\theta^{*} \approx 0.2-0.3$ corresponding to $50-100 \mathrm{kcal}$ for the combined reorganization energy.

The tunnelling factors are about $10^{-3}$ for $\mathrm{C}-\mathrm{C}$ stretching and $10^{-3}-10^{-2}$ for hydrogen atom transfer in insertion. These values were obtained from eqns. (34) and (35). Stretching frequencies of $1600 \mathrm{~cm}^{-1}$ and $1100 \mathrm{~cm}^{-1}$ for $\mathrm{C}-\mathrm{C}$ double and single bonds, respectively, and a bond length difference of $0.16 \AA$ were taken for the former while $600 \mathrm{~cm}^{-1}$ and $3000 \mathrm{~cm}^{-1}$, and a transfer distance of $0.7 \AA$ (Fig. 3), were taken for the hydrogen atom. The tunnelling probability for the hydrogen atom may be decreased at the expense of additional activation energy expendi- ture by compression and twisting of the Rhethylene unit. The fairly exothermic nature of the process also implies that kinetic deuterium isotope effects are not necessarily large even though tunnelling distances for hydrogen atoms are significant. ${ }^{32,33}$

The activation energy for bending of coordinated ethylene is finally calculated to be $25-30$ $\mathrm{kcal}$ if we use a bending frequency of $200 \mathrm{~cm}^{-1}$ representative of metal-ligand bending modes with a ligand mass corresponding to that of ethylene, and the bending angle is $25^{\circ}$ (Fig. 3 and Ref. 12b). Anharmonic effects are, however, undoubtedly important here due to very high vibrational excitation. The activation energy could thus be lowered by more than $10 \mathrm{kcal}$ by using for example a squared hyperbolic tangent potential $^{32}$ (the Morse-Rosen potential $^{34}$ ) and brought close to experimental values $^{29,31}$ and the value of $15-18 \mathrm{kcal}$ which emerges from SCF calculations. ${ }^{12 b}$

Extraction of rate parameters is less straightforward for oxidative addition due to the "mixing" of the nuclear modes and the nature of $\mathrm{Rh}-\mathrm{H}$ bending as intermediate between the classical and quantum limits. This mode is thus thermally excited to a certain vibrational level from which further distortion occurs by tunnelling. In order to specify as few of the inaccessible structural parameters as possible, estimates were made in the following way. $Q_{\mathrm{i}}^{\mathrm{s}^{*}}$ was calculated for different values of $\theta^{*}$ by means of eqn. (19). This also provides a value of $Q_{\mathrm{f}}^{\mathrm{b} *}$ from eqn. (21), and upper limits for the right hand side of eqn. (23). $\Omega_{\mathrm{f}}^{\mathrm{b}}$ was also here taken to be $600 \mathrm{~cm}^{-1}$, the $\mathrm{Rh}-\mathrm{H}$ bond length $1.65 \AA,{ }^{12 \mathrm{~b}}$ and $-\Delta U_{\mathrm{o}}>13 \mathrm{kcal}$. When the right hand side of eqn. (23) is compared with the left hand side for values of the reorganization energy terms in the range 5-15, $\mathrm{kcal}$, ignoring here the small value of the term which represents the mode $Q_{x}$, it is found that $\theta^{*} \approx 0.2-0.3$ are upper limits for solutions of eqn. (23). Larger values are thus only found for unrealistically large values of $E_{\mathrm{s}}+E_{\mathrm{r}}$.

This value of $\theta^{*}$ gives an upper limit of $1.15 \AA$ for the $\mathrm{H}_{2}$ bond length in the intermediate state corresponding to about $25 \%$ of the total change of this bond length when going from from the initial to the final state (Fig. 2). Using a vibrational frequency of $4000 \mathrm{~cm}^{-1}$ the tunnelling factor for $\mathrm{H}_{2}$ stretching is found to be $10^{-3}-0.1$, while the activation energy for $\mathrm{Rh}-\mathrm{H}$ bending is 
$10-15 \mathrm{kcal}$ if the bending frequency is again 600 $\mathrm{cm}^{-1}$ and the $\mathrm{Rh}-\mathrm{H}$ bond length $1.65 \AA$. The activation energy value would be lowered by $1-4$ kcal if we used an anharmonic potential such as the squared hyperbolic tangent potential. ${ }^{32}$

Eqns. (13), (24) and (32) - (35) finally provide indications as to the rate determining step of the overall reaction. In spite of the somewhat endothermic nature of the dissociation step $\left(\Delta U_{\mathrm{o}}>5-6 \mathrm{kcal}^{19}\right)$ this step represents rapid pre-equilibrium, largely because of low reorganization energies for the solvent and intramolecular modes. Both oxidative addition and insertion are accompanied by large restructuration, but in different nuclear parts, while differences in the solvent contributions are likely to be less important in view of the apolar nature of the reaction media and the small charge redistribution in the reacting complexes. Furthermore, since $E_{\mathrm{r}}$ and $\Delta U_{\mathrm{o}}$ are also similar, the difference between the activation parameters must be contributed largely by $\mathrm{H}_{2}$ stretching and $\mathrm{Rh}-\mathrm{H}$ bending in oxidative addition, and by Rh-ethylene-ethyl bending and $\mathrm{C}-\mathrm{C}$ stretching in insertion.

When we insert the activation energies and nuclear tunnelling factors estimated, in the rate expressions, it appears that the insertion step is rate determining primarily due to the large activation energy for bending of the coordinated olefin. The dominance of this particular mode to the activation energy of the insertion step also emerges from quantum chemical calculations of the energy changes which accompany the motion of the different molecular fragments in this step. ${ }^{12 b}$ The total rate difference between insertion and oxidative addition originating from the calculated activation energies and tunnelling factors seems, however, to be small enough that the latter step could be rate determining for other related systems.

\section{REFERENCES}

1. Sakaki, S., Kato, H., Hanai, H. and Tamara, K. Bull. Chem. Soc. Japan. 48 (1975) 813.

2. Novaro, O., Chow, S. and Magnonat, P. J. Catal. 41 (1976) 91.

3. Grima, J. P., Choplin, F. and Kaufmann, G. J. Organomet. Chem. 129 (1977) 221.

4. Lauher, J. W. and Hoffmann, R. J. Am. Chem. Soc. 98 (1976) 1729.
5. Fonnesbech, N., Hjortkjær, J. and Johansen, H. Int. J. Quant. Chem. 12 Suppl. 2 (1977) 95.

6. Novaro, O., Blaisten-Barojas, E., Clementi, E., Giunchi, S. and Ruiz-Vizcaya, M. E. $J$. Chem. Phys. 68 (1978) 2337.

7. Thorn, D. L. and Hoffmann, R. J. Am. Chem. Soc. 100 (1978) 2079.

8. Cassoux, P., Crasnier, F. and Labarre, J. F. J. Organomet. Chem. 165 (1979) 303.

9. McKinney, D. J. and Pensak, D. A. Inorg. Chem. 18 (1979) 3413.

10. Dedieu, A. and Strich, A. Inorg. Chem. 18 (1979) 2940.

11. McKinney, D. J. J. Chem. Soc. Chem. Commun. (1980) 490.

12. a. Dedieu, A. Inorg. Chem. 19 (1980) 375; b. 20 (1981) 2803.

13. Johansen, H. Chem. Phys. Lett. 84 (1981) 580.

14. Dogonadze, R. R. and Urushadze, Z. D. $J$. Electroanal. Chem. 32 (1971) 235.

15. a. Dogonadze, R. R., Kuznetsov, A. M. and Vorotyntsev, M. A. Phys. Status Solidi B 54 (1972) 125; b. 54 (1972) 425.

16. Dogonadze, R. R. and Kuznetsov, A. M. Prog. Surf. Sci. 5 (1975) 1.

17. Ulstrup, J. Charge Transfer Processes in Condensed Media, Lecture Notes in Chemistry, Springer, Berlin 1979, Vol. 10.

18. Ulstrup, J., In preparation.

19. Henrici-Olivé, G. and Olivé, S. Coordination and Catalysis, Verlag Chem., New York 1977.

20. James, B. R. Adv. Organomet. Chem. 17 (1979) 319.

21. Yared, Y. W., Miles, S. L., Bau, R. and Reed, C. A. J. Am. Chem. Soc. 99 (1977) 7076.

22. Meakin, P., Jesson, J. P. and Tolman, C. A. J. Am. Chem. Soc. 94 (1972) 3240.

23. Tolman, C. A., Meakin, P. Z., Lindner, D. L. and Jesson, J. P. J. Am. Chem. Soc. 96 (1974) 2762.

24. Masters, C. and Shaw, B. L. J. Chem. Soc. A (1971) 3679.

25. Van Gaal, H. L. M., Verlaak, J. M. J. and Posno, T. Inorg. Chim. Acta 23 (1977) 43.

26. German, E. D. and Dogonadze, R. R. J. Res. Inst. Catal. Hokkaido Univ. 20 (1972) 34.

27. German, E. D. and Dogonadze, R. R. Int. J. Chem. Kinet. 6 (1974) 457.

28. Vorotyntsev, M. A., Dogonadze, R. R. and Kuznetsov, A. M. Dokl. Akad. Nauk SSSR Ser. Fiz. Khim. 209 (1973) 1135. 
29. Osborn, J. A., Jardine, F. H., Young, J. F. and Wilkinson, G. J. Chem. Soc. A (1966) 1711.

30. Demortier, Y. and de Aguirre, I. Bull. Soc. Chim. Fr. (1974) 1673.

31. Rousseau, C., Evrard, M. and Petit, F. $J$. Mol. Catal. 3 (1977) 309.

32. Brüniche-Olsen, N. and Ulstrup, J. J. Chem. Soc. Faraday Trans. 1, 75 (1979) 205.

33. German, E. D. and Kuznetsov, A. M. $J$. Chem. Soc. Faraday Trans. 2, 77 (1981) 397.

34. Rosen, N. and Morse, P. Phys. Rev. 42 (1932) 210.

Received December 9, 1982. 